\title{
Inversions of Semistandard Young Tableaux
}

\author{
Paul Drube \\ Department of Mathematics and Statistics \\ Valparaiso University \\ Valparaiso, Indiana, U.S.A. \\ paul.drube@valpo.edu
}

Submitted: Aug 6, 2015; Accepted: Feb 17, 2016; Published: Mar 4, 2016

Mathematics Subject Classifications: 05A19, 05A05

\begin{abstract}
A tableau inversion is a pair of entries from the same column of a row-standard tableau that lack the relative ordering necessary to make the tableau columnstandard. An $i$-inverted Young tableau is a row-standard tableau with precisely $i$ inversion pairs, and may be interpreted as a generalization of (column-standard) Young tableaux. Inverted Young tableaux that lack repeated entries were introduced by Fresse to calculate the Betti numbers of Springer fibers in Type A, and were later developed as combinatorial objects in their own right by Beagley and Drube. This paper generalizes earlier notions of tableau inversions to row-standard tableaux with repeated entries, yielding an interesting new generalization of semistandard (as opposed to merely standard) Young tableaux. We develop a closed formula for the maximum numbers of inversion pairs for a row-standard tableau with a specific shape and content, and show that the number of $i$-inverted tableaux of a given shape is invariant under permutation of content. We then enumerate $i$-inverted Young tableaux for a variety of shapes and contents, and generalize an earlier result that places 1-inverted Young tableaux of a general shape in bijection with 0-inverted Young tableaux of a variety of related shapes.
\end{abstract}

Keywords: Young tableaux, inversions of Young tableaux

\section{Introduction}

Consider the non-increasing sequence of positive integers $\lambda=\left(\lambda_{1}, \lambda_{2}, \ldots, \lambda_{m}\right)$, and let $N=\lambda_{1}+\ldots+\lambda_{m}$. A Young diagram $Y$ of shape $\lambda$ is a left-justified array of $N$ total boxes such that there are $\lambda_{i}$ boxes in the $i^{\text {th }}$ row of $Y$. A (semistandard) filling of a Young diagram $Y$ is an assignment of positive integers (possibly repeated) to the boxes of $Y$ such that integers strictly increase from left-to-right across each row and weakly increase from top-to-bottom down each column. We assume that no positive integers are 
skipped, so that the boxes of $Y$ are filled with $1,2, \ldots, M$ for some $M \leqslant N$. We call the resulting array $T$ a semistandard Young tableau of shape $\lambda$. If each of $1,2, \ldots, N$ appears precisely once in $T$, the semistandard Young tableau $T$ qualifies as a standard Young tableau of shape $\lambda$. In this paper we will also need to consider a generalization of semistandard fillings where integers strictly increase from left-to-right across each row but no longer need to weakly increase down each column. We refer to such an array as a row-standard tableau.

If $\mu=\left(\mu_{1}, \mu_{2}, \ldots, \mu_{M}\right)$ is an ordered partition of $N$, we say that a semistandard tableau $T$ of shape $\lambda$ has content $\mu$ if its boxes are filled with precisely $\mu_{1}$ copies of $1, \mu_{2}$ copies of 2 , etc. We often use the abbreviated notation $\mu=1^{\mu_{1}} 2^{\mu_{2}} \ldots M^{\mu_{M}}$. Thus a standard Young tableau is simply a semistandard Young tableau with content $\mu=1^{1} 2^{1} \ldots N^{1}$. We denote the entire set of semistandard Young tableaux with shape $\lambda$ and content $\mu$ by $S(\lambda, \mu)$, and the set of standard Young tableaux with shape $\lambda$ by $S(\lambda)$. For a great introduction to Young tableaux, see Fulton [5].

Now consider the permutation $\sigma \in S_{n}$. An inversion of $\sigma$ is a pair of integers $i, j$ satisfying $i<j$ and $\sigma(i)>\sigma(j)$. In this situation we call $(i, j)$ an inversion pair of $\sigma$. Denote the number of distinct inversion pairs of $\sigma$ by $\mathrm{n}_{\text {inv }}(\sigma)$.

As introduced by Fresse in [3], permutation inversions admit a generalization to rowstandard tableaux with non-repeated entries. Let $Y$ be a Young diagram of shape $\lambda$ whose boxes have been filled with $1,2, \ldots, N$ to produce the row-standard tableau $\tau$. Following [3], a pair of entries $i, j$ from the same column of $\tau$ participate in an inversion of $\tau$ if $i<j$ and either of the following conditions hold:

1. At least one of $i$ and $j$ lacks an entry directly to its right, and $i$ is below $j$.

2. $i$ is directly to the left of $i^{\prime}, j$ is directly to the left of $j^{\prime}$, and $i^{\prime}>j^{\prime}$.

In this situation, we write $(i, j)_{\tau}$ or simply $(i, j)$ and say that $i, j$ constitute a single inversion pair of $\tau$. If a row-standard tableau $\tau$ has precisely $K$ distinct inversion pairs we write $\mathrm{n}_{\text {inv }}(\tau)=K$. Notice that a row-standard tableau $\tau$ is also column-standard and hence is a standard Young tableau if and only if $n_{\text {inv }}(\tau)=0$. Also notice that our definition of tableau inversion specializes to the earlier notion of permutation inversion if one interprets $\sigma$ as a single-column row-standard tableau whose entries appear as $\sigma(1), \ldots, \sigma(n)$ from top-to-bottom.

As shown in [3], for any row-standard tableau $\tau$ without repeated entries one can always recursively eliminate inversions to produce a unique column-standard tableau with no inversions. The resulting standard Young tableau is known as the standardization of $\tau$ and is written $\operatorname{st}(\tau)$. As any such $\tau$ may be transformed into a standard Young tableau st $(\tau)$ by recursively removing inversions, we henceforth refer to the row-standard $\tau$ as an inverted (standard) Young tableau based on st $(\tau)$. In Figure 1 we show an inverted tableau of shape $\lambda=(4,3,2)$ alongside its standardization. For a given shape $\lambda$, we denote the set of all inverted standard Young tableaux of shape $\lambda$ with precisely $i$ inversions by $S_{i}(\lambda)$. Thus $S(\lambda)=S_{0}(\lambda)$. We more specifically refer to elements of $S_{i}(\lambda)$ as i-inverted (standard) Young tableaux of shape $\lambda$. 


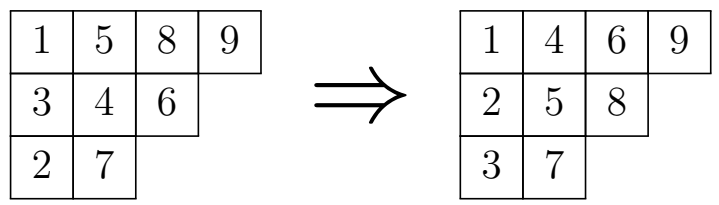

Figure 1: An inverted tableau with inversion pairs $(6,8),(1,3),(2,3)$ and its standardization

Fresse introduced tableau inversions in [3] to calculate the Betti numbers of Springer fibers in type A. Fixing the standard Young tableau $T$ of shape $\lambda$, he showed that the component of the Springer variety $F_{\lambda}$ associated with $T$ has $m^{\text {th }}$ Betti number equal to the number of $(d-m)$-inverted Young tableaux based on $T$, where $d$ is the dimension of the entire Springer variety. Fresse also presents an algorithm for determining the number of $i$-inverted Young tableaux based on a specific standard tableau $T$. In a more recent publication [4], Fresse, Mansour and Melnikov calculate the number of inverted tableaux in several special cases and show that the distribution of these numbers is unimodal.

In [1], the author and Beagley present results enumerating the total number of $i$ inverted Young tableaux of shape $\lambda$, simultaneously ranging over all underlying standardizations. By [3], this yielded easily calculable formulas for the Betti numbers of the entire Springer variety $F_{\lambda}$ in a number of interesting cases. In particular, [1] gives closed formulas for $\left|S_{1}(\lambda)\right|,\left|S_{M-1}(\lambda)\right|$, and $\left|S_{M-2}(\lambda)\right|$, where $M$ is the maximum number of inversions possible for any inverted Young tableau of shape $\lambda$. That same paper also presents closed formulas for general $\left|S_{i}(\lambda)\right|$ in the case of relatively "easy" choices for $\lambda$.

The combinatorial results of [1] also formalized earlier work on the Bar-Natan skein module of the solid torus presented by Russell in [6], with the generators of Russell's skein module standing in bijection with inverted Young tableaux of shape $\lambda=(n, n)$. It is hypothesized that Russell's work extends to the $s l_{n}$ skein module of the solid torus for all $n \geqslant 2$, giving an interesting topological interpretation of inverted Young tableaux for any rectangular shape $\lambda=(n, \ldots, n)$. An upcoming paper by the author [2] explicitly demonstrates this correspondence in the $n=3$ case.

The primary goal of this paper is to generalize the notion of tableau inversions to the semistandard case, where repeated entries are possible, and to investigate which results from [3] and [1] extend to this more sophisticated case. Although the algebraic geometry of this case has not been explicitly worked out, seeing as Spaltenstein varieties are the generalization of Springer varieties corresponding to semistandard Young tableaux, the author suspects that this paper may shed light on the Betti numbers of Spaltenstein varieties. In the spirit of [6] and [2], the author also suspects that this semistandard generalization will be topologically realized by skein modules of the solid torus where the boundary circles are not consistently oriented. Do note that the focus of this paper is purely combinatorial; no knowledge of algebraic varieties or skein modules is required, and Springer/Spaltenstein varieties will only be mentioned in passing.

So let $\tau$ be a row-standard tableau of shape $\lambda$ and content $\mu$, and let $i, j$ be a pair of entries from the same column of $\tau$. Let $\left\{i_{1}, i_{2}, \ldots\right\}$ denote the (possibly empty) sequence 
of entries directly to the right of $i$ in $\tau$, read from left-to-right, and let $\left\{j_{1}, j_{2}, \ldots\right\}$ denote the (possibly empty) sequence of entries directly to the right of $j$, read from left-to-right. We assert that $i, j$ participate in an inversion of $\tau$ if $i<j$ and one of the following holds:

1. At least one of $i_{1}$ and $j_{1}$ doesn't exist, and $i$ is below $j$.

2. $i_{1}$ and $j_{1}$ both exist, and $i_{1}>j_{1}$.

3. $i_{k}$ and $j_{k}$ both exist for all $k \leqslant M$ with $i_{k}=j_{k}$ for all $k \leqslant M$, at least one of $i_{M+1}$ or $j_{M+1}$ doesn't exist, and $i$ is below $j$.

4. $i_{k}$ and $j_{k}$ both exist for all $k \leqslant M$ with $i_{k}=j_{k}$ for all $k \leqslant M, i_{M+1}$ and $j_{M+1}$ both exist, and $i_{M+1}>j_{M+1}$.

In Section 2, the somewhat redundant definition above will be streamlined utilizing what we refer to as the "height order" on tableau entries. The reason for the lengthier set of conditions above is that it betrays how our notion is a direct generalization of tableau inversions for standard tableaux: as $i_{k}=j_{k}$ is impossible in the case of nonrepeated entries, only the first two conditions above are relevant in that situation. If any of the conditions above hold, we once again write $(i, j)_{\tau}$ or simply $(i, j)$ and say that $i, j$ constitute an inversion pair of $\tau$. We also retain our notation that $\mathrm{n}_{\text {inv }}(\tau)$ denotes the total number of distinct inversion pairs in $\tau$. In this case, the row-standard $\tau$ qualifies as a semistandard Young tableau if and only if $n_{\text {inv }}(\tau)=0$.

A direct generalization of the technique from [3] shows that one may recursively remove inversions in any row-standard tableau $\tau$ to produce a column-standard semistandard tableau with no inversions, which we again refer to as the standardization $\operatorname{st}(\tau)$ of $\tau$. This standardization is merely the semistandard tableau where one has independently reordered the entries in each column so that they are weakly-increasing from top-tobottom, and is guaranteed to be row-standard if the original $\tau$ was row-standard. This fact prompts our definition of $\tau$ as an inverted semistandard Young tableau based on $\operatorname{st}(\tau)$. Figure 2 shows an example of an inverted semistandard tableau with $\lambda=(4,4,3)$ and $\mu=1^{1} 2^{1} 3^{1} 4^{1} 5^{2} 6^{1} 7^{2} 8^{2}$. If $\mathrm{n}_{\text {inv }}(\tau)=i$, we refer to $\tau$ as an $\mathbf{i}$-inverted semistandard Young tableau. For given $\lambda$ and $\mu$, we denote the set of all such tableaux with precisely $i$ inversion pairs by $S_{i}(\lambda, \mu)$. If we range across all possible numbers of inversions, we collectively refer to the set of all inverted semistandard tableaux as $I(\lambda, \mu)=\bigcup_{i=0}^{\infty} S_{i}(\lambda, \mu)$.

\begin{tabular}{|l|l|l|l}
\hline 1 & 3 & 7 & 8 \\
\hline 4 & 5 & 6 & 8 \\
\hline 2 & 5 & 7 & $\Rightarrow$
\end{tabular}$\Rightarrow$\begin{tabular}{ll|l|l|l|}
\hline 1 & 3 & 6 & 8 \\
\hline 2 & 5 & 7 & 8 \\
\hline 4 & 5 & 7 & \multicolumn{1}{|c}{} \\
\cline { 1 - 3 }
\end{tabular}

Figure 2: An inverted semistandard tableau with inversion pairs $(2,4),(3,5),(6,7)$ and its standardization.

It should be noted that the notion of "tableau inversion" presented here as well as in [3],[1] is a distinct concept from the "inversions in standard Young tableaux" introduced 
by Shynar in [7]. In [7], a (weak) inversion in a standard Young tablau $T$ is a pair $(i, j)$ of entries such that $i<j$ and where $j$ appears both strictly south and strictly (resp. weakly) west of $j$ in $T$. As such, Synar's inversions are a measure on standard Young tableau that do not address the more general row-standard case. Although possibly related to the maximal possible number of inversion pairs in an inverted Young tableau $\tau$ with $\operatorname{st}(\tau)=T$, Shynar's distinct notion of tableau inversion will have absolutely no bearing on what follows.

\subsection{Outline of Results}

We begin in Section 2 by generalizing a variety of basic results from [1] to the semistandard case. Our most significant theorem in this realm is a closed formula giving the maximum possible number of inversion pairs for an inverted semistandard Young tableau of given shape and content, a result that eventually appears as Theorem 7 and is presented in truncated form below:

Theorem 1. Consider the shape $\lambda=\left(\lambda_{1}, \ldots, \lambda_{m}\right)$ and content $\mu=1^{\mu_{1}} 2^{\mu_{2}} \ldots K^{\mu_{K}}$, and let $h_{j}$ be the height of the $j^{\text {th }}$ column in any tableau of shape $\lambda$. If $I(\lambda, \mu)$ is nonempty, then the maximum number of inversions for any element of $I(\lambda, \mu)$ is:

$$
M_{\lambda, \mu}=\sum_{j}\left(\begin{array}{c}
h_{j} \\
2
\end{array}\right)-\sum_{j}\left(\begin{array}{c}
\mu_{i} \\
2
\end{array}\right)
$$

Also included in Section 2 is the most theoretically significant result of the paper, a demonstration that the number of $i$-inverted semistandard Young tableaux of a fixed shape is invariant under "permutation of content". Eventually appearing as Theorem 11, notice that the simplified version shown below specializes to the well-known invariance of semistandard Young tableau presented in [5] and elsewhere if we let $i=0$ :

Theorem 2. Take any shape $\lambda$ and any content $\mu$ using the entries $1,2, \ldots, M$. For any permutation $\sigma$ on $M$ letters, we have $\left|S_{i}(\lambda, \mu)\right|=\left|S_{i}(\lambda, \sigma(\mu))\right|$ for all $i \geqslant 0$.

Section 3 proceeds to give a series of direct enumerative results about inverted semistandard Young tableaux. Closed formulas are given for the number of $i$-inverted tableaux in the one-column case (Theorem 13) and two-row case (Theorem 14), for any valid content $\mu$. Our result for the one-column case, which reveals an intriguing new application of the q-factorial, is shown below:

Theorem 3. Let $\lambda$ be the one-column tableau shape with $M$ total entries, and let $\mu=$ $1^{\mu_{1}} 2^{\mu_{2}} \ldots m^{\mu_{m}}$ be any content with $\sum_{k} \mu_{k}=M$. Then the $\left|S_{i}(\lambda, \mu)\right|$ have generating function:

$$
\sum_{i=0}^{\infty}\left|S_{i}(\lambda, \mu)\right| q^{i}=\frac{[M]_{q} !}{\left[\mu_{1}\right]_{q} !\left[\mu_{2}\right]_{q} ! \ldots\left[\mu_{m}\right]_{q} !}
$$

Where $[p]_{q}=1+q+\ldots+q^{p-1}$ is the q-number and $[p]_{q} !=[p]_{q}[p-1]_{q} \ldots[2]_{q}[1]_{q}$ is the $q$-factorial. 
We close the paper with proofs of Theorems 16 and 17, which directly generalize results from [1] by demonstrating a bijection between 1-inverted semistandard Young tableaux of a given shape and 0-inverted semistandard Young tableaux of a collection of related shapes. The more easily-digested specialization of this result to rectangular shapes is given below:

Theorem 4. Let $m, n \geqslant 1$, and take the $m$-row tableau shapes $\lambda=(n, \ldots, n), \tilde{\lambda}=$ $(n+1, n, \ldots, n, n-1)$. Then $\left|S_{1}(\lambda, \mu)\right|=\left|S_{0}(\lambda, \mu)\right|$ for any content $\mu$ compatible with $\lambda$.

It should be reiterated that the focus of this paper is purely combinatorial. Many of the results above suggest reinterpretations in terms of algebraic varieties if the terminology is carefully recast. For example, Theorem 3 may be interpreted as an equality between two writings of the Poincare polynomial for the variety $\mathcal{F}$ of partial flags $V_{0}=0 \subset V_{1} \subset$ $\ldots \subset V_{m}=\mathbb{C}^{M}$ with $\operatorname{dim}\left(V_{i} / V_{i-1}\right)=\mu_{i}$. Induction shows that the right-side of Theorem 3 directly equals to the Poincare polynomial of $\mathcal{F}$, whereas the $\left|S_{i}(\lambda, \mu)\right|$ on the left-side equal the number of $i$-dimensional cells in the Bruhat decomposition of $\mathcal{F}$.

\section{Basic Results About Inverted Semistandard Young Tableaux}

Before moving on to specific results about inverted semistandard Young tableaux, we formalize the definition of tableau inversion from Section 1 via a complete order on the entries of any fixed column in a tableau. So let $\tau$ be an inverted semistandard Young tableau. Beginning with the rightmost column of $\tau$ and recursively working our way leftward, we place a complete order 4 on the entries $\left\{a_{i}\right\}$ of each column as follows:

- If either $a_{i}$ or $a_{j}$ lacks an entry directly to its right and $a_{i}$ lies above $a_{j}$, then $a_{i} \triangleleft a_{j}$.

- If $a_{i}$ lies directly to the left of $b_{i}, a_{j}$ lies directly to the left of $b_{j}$, and $b_{i}<b_{j}$, then $a_{i} \triangleleft a_{j}$.

- If $a_{i}$ lies directly to the left of $b_{i}, a_{j}$ lies directly to the left of $b_{j}, b_{i}=b_{j}$, and $b_{i} \boldsymbol{4} b_{j}$, then $a_{i}<a_{j}$.

We call $\varangle$ the height order on the $j^{\text {th }}$ column of $\tau$. If $c$ is the $k^{\text {th }}$ smallest element in its column of $\tau$ relative to the height order on that column, we say that $c$ has a height of $k$ in $\tau$ and write $\operatorname{ht}(c)=k$.

The order $\varangle$ tells us how a column of a tableau "should be" ordered (relative to the column immediately on its right) if that column is to avoid any inversion pairs. Notice that, if $\tau$ is column semistandard, then the height of $c$ is always equal to its row number. In an inverted tableau, if ht $(c)$ does not equal the row number of $c$, then $c$ is involved in at least one inversion pair. Most generally:

Proposition 5. Let $\tau$ be a row-standard tableau and let $i, j$ be two entries from the same column of $\tau$. Then $(i, j)$ forms an inversion pair of $\tau$ if and only if $i<j$ and $j \varangle i$. 
As one final basic comment about inversion pairs notice that, unlike in the nonrepeated entry case of [1] and [3], the location of an inversion pair $(i, j)$ is not uniquely identified by specifying which two entries are involved. This is because, when one allows for repeated entries, it is possible for a pair of entries to appear together in more than one column of an inverted tableau or even for a specific inversion $(i, j)$ to appear multiple times in one column (if different instances of $i$ and $j$ are involved in those distinct inversions). When one needs to specify the column of origin for an inversion pair, we henceforth use $(i, j)^{k}$ to denote that the inversion pair $(i, j)$ occurs in the $k^{t h}$ column of $\tau$. Much as a standardization $T$ and a collection of inversion pairs was enough to uniquely identify a particular inverted standard Young tableau $\tau$ in the setting of [3] of [1], it is straightforward to show that a standardization along with a collection of column-specified inversion pairs (with multiplicities for each column) is enough to uniquely identify a particular inverted semistandard Young tableau.

For the remainder of this section, we consider which basic results about inverted standard Young tableau from [1] generalize to the semistandard case. The most fundamental result discussed in [1] was an explicit formula for the total number of inverted Young tableaux $|I(\lambda)|$ of an arbitrary shape $\lambda=\left(\lambda_{1}, \lambda_{2}, \ldots, \lambda_{m}\right)$. A quick counting argument yielded:

$$
|I(\lambda)|=\left(\begin{array}{c}
\lambda_{1}+\ldots+\lambda_{m} \\
\lambda_{m}
\end{array}\right)\left(\begin{array}{c}
\lambda_{1}+\ldots+\lambda_{m-1} \\
\lambda_{m-1}
\end{array}\right) \ldots\left(\begin{array}{c}
\lambda_{1}+\lambda_{2} \\
\lambda_{2}
\end{array}\right)=\frac{\left(\lambda_{1}+\ldots+\lambda_{m}\right) !}{\lambda_{1} ! \lambda_{2} ! \ldots \lambda_{m} !}
$$

Unfortunately, Equation 1 does not appear to possess a tractable generalization to the general semistandard case $|I(\lambda, \mu)|$. In particular, the necessity of the row-standard condition with regard to repeated entries prompts a series of increasingly sophisticated sub-cases and prevents a succinct probabilistic formulation akin to the rightmost side of Equation 1. One of the few specific cases where $|I(\lambda, \mu)|$ is directly calculable with our current resources is when $\lambda$ has one column:

Proposition 6. Let $\lambda=1^{M}$ be the one-column tableau shape with $M$ total entries, and let $\mu=1^{\mu_{1}} 2^{\mu_{2}} \ldots m^{\mu_{m}}$ be some content such that $\sum_{k} \mu_{k}=M$. Then $|I(\lambda, \mu)|=$ $M !$ $\overline{\mu_{1} ! \mu_{2} ! \ldots \mu_{m} !}$.

Proof. Temporarily assume that all of the entries are distinct. In this case there are $M$ ! possible arrangements. Dividing through by $\mu_{i}$ ! then accounts for the fact that the $\mu_{i}$ instances of $i$ are indistinguishable, thus accounting for repetitions in our original enumeration.

Luckily, the remaining results from Chapter 2 of [1] all admit generalizations to semistandard tableaux. In Subsection 2.1 we prove a general formula for the "maximum inversion number" of an element in $I(\lambda, \mu)$. In Subsection 2.2 we then prove an extremely useful result about the invariance of the $\left|S_{i}(\lambda, \mu)\right|$ under permutation of content: a theorem that has no analog in [1] but which directly generalizes the classic permutation invariance 
of (non-inverted) semistandard Young tableaux. All enumerative results, including the "straightforward cases" of one-column and two-row tableaux, are delayed until Section 3 so that they can make direct usage of the permutation invariance of Theorem 11.

\subsection{Maximum Number of Inversions for Shape $\lambda$ and Content $\mu$}

Obviously, a tableau of finite size cannot possess an infinite number of inversions. It is then of interest to ask the maximum number of inversion pairs that an element of $I(\lambda, \mu)$ may possess. In other words, what is the largest $i$ for which $\left|S_{i}(\lambda, \mu)\right|$ is nonempty? For an inverted tableau without repeated entries, in [1] it was shown that the maximum such $i$ for an element of $I(\lambda)$ was:

$$
M_{\lambda}=\sum_{j} T_{\left(h_{j}-1\right)}=\sum_{j}\left(\begin{array}{c}
h_{j} \\
2
\end{array}\right)
$$

where $T_{k}=1+2+\ldots+k$ is the triangle number and $h_{j}$ is the height of the $j^{\text {th }}$ column in any tableau of shape $\lambda$. In addition to an explicit formula for $M_{\lambda},[1]$ also showed that there was always precisely one element in $I(\lambda)$ with $M_{\lambda}$ inversions. When one allows for general content $\mu$ with repeated entries, Equation 2 directly generalizes to the following:

Theorem 7. Let $\lambda=\left(\lambda_{1}, \ldots, \lambda_{m}\right)$ and $\mu=1^{\mu_{1}} 2^{\mu_{2}} \ldots K^{\mu_{K}}$, and define $h_{j}=\left|\left\{\lambda_{i} \mid \lambda_{i} \geqslant j\right\}\right|$ to be the height of the $j^{\text {th }}$ column for any tableau of shape $\lambda$. If $I(\lambda, \mu)$ is nonempty, then the maximum number of inversions for any inverted semistandard Young tableau of shape $\lambda$ with content $\mu$ is:

$$
M_{\lambda, \mu}=\sum_{j} T_{\left(h_{j}-1\right)}-\sum_{i} T_{\left(\mu_{i}-1\right)}=\sum_{j}\left(\begin{array}{c}
h_{j} \\
2
\end{array}\right)-\sum_{i}\left(\begin{array}{c}
\mu_{i} \\
2
\end{array}\right)
$$

Moreover, this maximum inversion number is realized by precisely one inverted semistandard Young tableau of shape $\lambda$ and content $\mu$, so that $\left|S_{M_{\lambda, \mu}}(\lambda, \mu)\right|=1$.

Proof. Our strategy is to pick an arbitrary semistandard Young tableau $T \in S(\lambda, \mu)$ and argue that an inverted tableau $\tau \in I(\lambda, \mu)$ with $\operatorname{st}(\tau)=T$ can have no more than $\sum_{j} T_{\left(h_{j}-1\right)}-\sum_{i} T_{\left(\mu_{i}-1\right)}=M_{\lambda, \mu}$ inversions pairs. We then show that there is a unique semistandard tableau $T^{*} \in S(\lambda, \mu)$ such that an inverted tableau with $\operatorname{st}(\tau)=T^{*}$ can actually obtain that upper bound, and we construct a unique inverted tableau $\tau_{\max }$ with standardization $T^{*}$ such that $\mathrm{n}_{\text {inv }}\left(\tau_{\max }\right)=M_{\lambda, \mu}$.

So fix $\lambda, \mu$, and let $h_{j}$ denote height of the $j^{\text {th }}$ column in any tableau of shape $\lambda$. Then take any semistandard tableau $T \in S(\lambda, \mu)$, and pick a value $\alpha$ in $T$. As $T$ is row-standard, at most one copy of $\alpha$ may appear in each row of $T$. This allows us to place a complete order $\prec$ on the $\mu_{\alpha}$ copies of $\alpha$ in $T$ such that $\alpha_{k} \prec \alpha_{l}$ if $\alpha_{k}$ appears in a higher row than $\alpha_{l}$. Henceforth index the copies of $\alpha$ in $T$ according to this complete order, so that $\alpha_{1} \prec \ldots \prec \alpha_{\mu_{\alpha}}$. Pause to notice that, since $T$ is column semistandard, $\alpha_{k} \prec \alpha_{l}$ ensures that $\alpha_{k}$ appears at least as far right in $T$ as does $\alpha_{l}$. 
Now consider the $j^{\text {th }}$ column of $T$, whose entries we denote $a_{1} \leqslant a_{2} \leqslant \ldots \leqslant a_{h_{j}}$ from top to bottom. This means that $a_{i}$ has height $\operatorname{ht}_{T}\left(a_{i}\right)=i$ in $T$. If we take any $\tau \in I(\lambda, \mu)$ with $\operatorname{st}(\tau)=T$, we can immediately say that the $j^{t h}$ column of $\tau$ may possess at most $(i-1)$ inversion pairs in which $a_{i}$ is the larger entry. ${ }^{1}$ Ranging over all $a_{i}$, we see that the $j^{\text {th }}$ column of $\tau$ may possess at most $T_{\left(h_{j}-1\right)}$ inversion pairs, allowing use to conclude that $\tau$ may contain at most $\sum_{j} T_{\left(h_{j}-1\right)}$ inversion pairs (see Equation 2).

To obtain the more precise upper bound of this theorem, let $b_{1} \leqslant b_{2} \leqslant \ldots \leqslant b_{h_{j+1}}$ denote the entries in the $(j+1)^{s t}$ column of $T$, read from top to bottom. For a fixed entry $a_{i}$ in the $j^{\text {th }}$ column of $T$, let $b_{\gamma_{i}}$ be the highest entry in the $(j+1)^{s t}$ column of $T$ such that $b_{\gamma_{i}}>a_{i}$, so that there are precisely $\gamma_{i}-1$ entries $b_{k}$ in the $(j+1)^{s t}$ column of $T$ with $b_{k} \leqslant a_{i}$. For any $\tau$ with $\operatorname{st}(\tau)=T$, notice that row-standardness ensures that $\operatorname{ht}_{\tau}\left(a_{i}\right) \geqslant \gamma_{i}$ in $\tau$ and hence that the $j^{\text {th }}$ column of $\tau$ may contain at most $i-\gamma_{i}$ inversion pairs whose larger entry is $a_{i}$. We are now ready to account for repeated entries.

So assume that an entry $a_{i}$ in the $j^{\text {th }}$ column of $T$ is the $k^{\text {th }}$ instance of $\alpha$ according to our complete order $\prec$, namely $a_{i}=\alpha_{k}$. Additionally assume there are $k_{1}$ other instances of $\alpha$ above $a_{i}$ in the $j^{\text {th }}$ column of $T$ and there are $k_{2}$ instances of $\alpha$ in a more rightward column of $T$, so that $k_{1}+k_{2}=k-1$. It follows that there are at least $k_{2}$ entries in the $(j+1)^{s t}$ column of $T$ that are smaller than $a_{i}$, so that $\gamma_{i} \geqslant k_{2}+1$. As flipping identical entries fails to produce an inversion pair, there are $k_{1}$ additional entries above $a_{i}$ in $T$ with which $a_{i}$ may not form an inversion pair in any $\tau$ with $\operatorname{st}(\tau)=T$. It follows that the $j^{\text {th }}$ column of $\tau$ may contain at most $i-\left(k_{2}+1\right)-k_{1}=i-k$ inversion pairs whose larger entry is $a_{i}$. Notice that this "inversion deficiency" for $a_{i}$, when compared to the non-repeated entry case of Equation 2, is $k-1$. So when we range over all instances of $\alpha$ in $\tau$ we must have at least $0+1+\ldots+\left(\mu_{\alpha}-1\right)=T_{\left(\mu_{\alpha}-1\right)}$ fewer inversions than the maximum number of inversion pairs allowed by Equation 2. Ranging over all values $\alpha$ allows us to conclude that any $\tau$ with $\operatorname{st}(\tau)=T$ may have at most $M_{\lambda, \mu}=\sum_{j} T_{\left(h_{j}-1\right)}-\sum_{i} T_{\left(\mu_{i}-1\right)}$ inversions.

Now consider the unique $T^{*} \in S(\lambda, \mu)$ such that, for all $j$, every entry in the $(j+1)^{s t}$ column of $T^{*}$ is at least as large as every entry in the $j^{\text {th }}$ column of $T^{*}$. We claim that this is the only tableau in $S(\lambda, \mu)$ such that $\tau \in I(\lambda, \mu)$ with $\operatorname{st}(\tau)=T^{*}$ may possess up to $M_{\lambda, \mu}$ inversion pairs. So let $T$ be any other semistandard tableau in $S(\lambda, \mu)$. For some $j$, there exists entries $a_{i}$ in the $j^{\text {th }}$ column of $T$ and $b$ in the $(j+1)^{s t}$ column of $T$ such that $a_{i}>b$. If $a$ is the $k^{t h}$ instance of $\alpha$ in our complete order $\prec$, equivalent reasoning to the previous paragraph ensures that any $\tau \in I(\lambda, \mu)$ with $\operatorname{st}(\tau)=T$ may contain at most $i-k-1$ inversion pairs in its $j^{\text {th }}$ column whose larger entry is $a_{i}$. As our previously-established upper bounds are unaffected for all other entries in $T$, we may conclude that any $\tau$ with $\operatorname{st}(\tau)=T$ may contain at most $M_{\lambda, \mu}-1$ inversion pairs.

It remains to be shown that there is precisely one $\tau \in I(\lambda, \mu)$ with $\operatorname{st}(\tau)=T^{*}$ such that $\mathrm{n}_{\mathrm{inv}}(\tau)=M_{\lambda, \mu}$. We begin by constructing the requisite tableau, which we call $\tau_{\max }$. Working one column at a time, from right-to-left, we place the $h_{n}$ largest available entries in the rightmost $\left(n^{\text {th }}\right)$ column of $\tau_{\max }$ from top-to-bottom in the unique nonincreasing order. For the $(n-1)^{s t}$ column, we work through the $h_{n-1}$ largest remaining

\footnotetext{
${ }^{1}$ Throughout this proof, $a_{i}$ refers to a specific copy of an entry $\alpha$, so that if the $j^{\text {th }}$ column contains multiple copies $\alpha$ there may be more than $i-1$ total inversion pairs in the $j^{t h}$ column of the form $\left(a_{k}, \alpha\right)$.
} 
entries in decreasing order, placing each element in the available spot with the lowest height that does not violate the row-standard condition. Notice that, if the instances of a repeated entry are split across two columns, this means that an entry need not be placed in the available spot with the lowest height. Repeat this procedure for each of the remaining columns, placing the largest remaining entry in the lowest height slot available that does not result in identical entries being placed in the same row. As we are recursively placing smaller entries leftward, the resulting tableau $\tau_{\max }$ is clearly row-standard and has standardization $\operatorname{st}\left(\tau_{\max }\right)=T^{*}$. For an example of the resulting $\tau_{\max }$, see Figure 3 .

\begin{tabular}{|l|l|l|}
\hline 1 & 4 & 5 \\
\hline 2 & 3 & 5 \\
\hline 2 & 3 &
\end{tabular}$\Longrightarrow$\begin{tabular}{l|l|l|l|}
\hline 1 & 2 & 5 \\
\hline 1 & 2 \\
\cline { 1 - 1 } 1 & 3 & 5 \\
\hline 2 & 3 & \multicolumn{1}{|c}{} \\
\cline { 1 - 2 } 2 & 4 &
\end{tabular}

Figure 3: The unique inverted semistandard Young tableau $\tau_{\max }$ for $\lambda=(3,3,2,2)$ and $\mu=1^{2} 2^{3} 3^{2} 4^{1} 5^{2}$ with $M_{\lambda, \mu}=7$ inversion pairs, alongside its standardization $T$.

The tableau $\tau_{\max }$ has been specifically constructed so that it maximizes the number of inversion pairs involving each element upon the placement of that element. In particular, assume that the $i^{\text {th }}$ entry placed in the $j^{\text {th }}$ column of $\tau_{\text {max }}$, namely $a_{i}$, is the $k^{\text {th }}$ instance of the value $\alpha$ to have been placed in $\tau_{\max }$. We consider two cases, depending upon whether the instances of $\alpha$ are split across one of two columns of $\tau_{\max }$. If all previous instances of $\alpha$ also lie in the $j^{\text {th }}$ column of $\tau_{\max }$, there are $i-(k-1)$ strictly smaller entries $a<a_{i}$ in the $j^{\text {th }}$ column, and we have $a_{i} \triangleleft a$ for all such $a$. If $k_{1}$ instances of $\alpha$ had previously been placed in the $j^{\text {th }}$ column and $k_{2}$ instances of $\alpha$ had previously been placed in the $(j+1)^{s t}$ column (so that $k_{1}+k_{2}=k-1$ ), there are $i-k_{1}$ strictly smaller entries $a<a_{i}$ in the $j^{\text {th }}$ column but $k_{2}$ of those entries (those directly to the left of other instances of $\alpha$ ) have $a \triangleleft a_{i}$. This leaves $i-k_{1}-k_{2}$ other entries $a$ in the $j^{\text {th }}$ column such that $a<a_{i}$ and $a_{i}<a$. In both cases, we may conclude that $\tau_{\max }$ contains precisely $i-(k-1)$ inversion pairs in which $a_{i}$ is the larger element. This gives $\tau_{\text {max }}$ a total of $\mathrm{n}_{\mathrm{inv}}(\tau)=M_{\lambda, \mu}$ inversion pairs when ranging over all entries $a_{i}$ and all columns.

Lastly, we claim that $\tau_{\max }$ is the only inverted tableau with standardization $T^{*}$ that may possess $M_{\lambda, \mu}$ inversion pairs. If we take any other $\tau \in I(\lambda, \mu)$ such that $\operatorname{st}(\tau)=T^{*}$, $\tau$ is related to $\tau_{\max }$ in that at least one of its columns have been re-ordered. So assume that the $j^{\text {th }}$ column of $\tau$ has a distinct ordering from $\tau_{\max }$. Via our construction of $\tau_{\max }$, there must exists an entry $a_{i}$ in the $j^{\text {th }}$ column of $\tau$ such that, if $a_{i}$ corresponds to the $k^{\text {th }}$ instance of $\alpha$ in our complete ordering $\prec$ on $T^{*}$, there exists fewer than $\operatorname{ht}\left(a_{i}\right)-k-1$ inversion pairs in the $j^{\text {th }}$ column of $\tau$ whose larger entry is $a_{i}$. As our previously-argued upper bounds for inversions involving other elements still holds, we conclude that $\tau$ has at most $M_{\lambda, \mu}-1$ total inversions. Thus $\tau_{\max }$ is the only inverted tableau with standardization $T^{*}$, and by extension the only element of $I(\lambda, \mu)$, with precisely $M_{\lambda, \mu}$ inversion pairs. 


\subsection{Invariance Under Permutation of Content}

One of the most fundamental results involving semistandard Young tableaux is that the number of such tableaux with a fixed shape $\lambda$ is invariant under permutation of content. In particular, given a content $\mu=\left(\mu_{1}, \mu_{2}, \ldots, \mu_{M}\right)$ and any permutation $\sigma \in S_{M}$, then $|S(\lambda, \mu)|=|S(\lambda, \sigma(\mu))|$. The most common proof of that fact, as outlined in [5], identifies the number of semistandard tableaux of given content as the coefficient in a certain Schur polynomial and then utilizes the fact that Schur polynomials are symmetric polynomials.

In this Subsection we show that the "permutation invariance" outlined above extends to i-inverted semistandard tableaux with a fixed number of inversions: that $\left|S_{i}(\lambda, \mu)\right|=$ $\left|S_{i}(\lambda, \sigma(\mu))\right|$ for all $i \geqslant 0$. Since the traditional notion of semistandard Young tableau corresponds to the case of $i=0$, our general result specializes to the previously-established permutation invariance result of [5] when $i=0$. Note that our techniques in no way reference symmetric polynomials, meaning that our $i=0$ specialization offers an apparently new proof of the well-known result from [5].

Before proceeding to our primary proof, we require a series of technical lemmas characterizing how inversion numbers behave under manipulations of inverted semistandard Young tableaux with certain "basic" shapes.

Lemma 8. Let $\tau$ be a one-column row-standard tableau with $N$ total boxes and content $\mu=1^{j} 2^{N-j}$. If $\tau^{*}$ is the row-standard tableau of content $\mu$ obtained by reversing the vertical ordering of $\tau$, then $\mathrm{n}_{\mathrm{inv}}(\tau)+\mathrm{n}_{\mathrm{inv}}\left(\tau^{*}\right)=j(N-j)$.

Proof. Notice that the maximum possible number of inversions for a tableau with given $\lambda$ and $\mu$ is $j(N-j)$, occurring when all 2 entries lie above all 1 entries. Now take any two entries $a_{i}, a_{j}$ in $\tau$ such that $a_{i} \neq a_{j}$. The entries $a_{i}$ and $a_{j}$ form an inversion pair in $\tau$ if and only if their reflections $a_{N-i+1}, a_{N-j+1}$ do not form an inversion pair in $\tau^{*}$. It follows that any such pair $a_{i}, a_{j}$ constitutes an inversion pair in precisely one of $\tau$ or $\tau^{*}$. Thus $\mathrm{n}_{\text {inv }}(\tau)+\mathrm{n}_{\text {inv }}\left(\tau^{*}\right)=j(N-j)$.

Lemma 9. Let $\tau$ be a one-column row-standard tableau with $N$ total boxes and content $\mu=1^{j} 2^{N-j}$. If $\bar{\tau}$ is the row-standard tableau of content $\bar{\mu}=1^{N-j} 2^{j}$ obtained by flipping all instances of 1 and 2 in $\tau$, then $\mathrm{n}_{\text {inv }}(\tau)+\mathrm{n}_{\text {inv }}(\bar{\tau})=j(N-j)$.

Proof. Notice that the maximum possible number of inversions for a tableau with given $\lambda$ and either content $\mu$ or $\bar{\mu}$ is $j(N-j)$, once again occurring when all 2 entries lie above all 1 entries. Take any two entries $a_{i}, a_{j}$ in $\tau$ such that $a_{i} \neq a_{j}$, and let $\bar{a}_{i}, \bar{a}_{j}$ be the equivalently placed entries in $\bar{\tau}$. The entries $a_{i}$ and $a_{j}$ form an inversion pair in $\tau$ if and only if $\bar{a}_{i}$ and $\bar{a}_{j}$ do not form an inversion pair in $\bar{\tau}$, as the relative ordering of the entries has been inverted in $\bar{\tau}$. It follows that $\mathrm{n}_{\text {inv }}(\tau)+\mathrm{n}_{\text {inv }}(\bar{\tau})=j(N-j)$.

Lemma 10. Let $\lambda$ be a two-column tableau of shape $\lambda$ with $N$ total boxes. If we define contents $\mu=1^{j} 2^{N-j}$ and $\bar{\mu}=1^{N-j} 2^{j}$, where $0 \leqslant j \leqslant N$, then $\left|S_{i}(\lambda, \mu)\right|=\left|S_{i}(\lambda, \bar{\mu})\right|$ for every $i \geqslant 0$. 
Proof. The general form of such a row-standard tableau (with content $\mu$ or $\bar{\mu}$ ) is shown in Figure 4. No matter the number of inversions, the only portion of such a tableau that is not determined is the one-column "tail". Observe that any inversion pairs from such a tableau must occur in its "tail". For any $\tau \in I(\lambda, \mu)$, we refer to the two-column "head" subtableau as $\tau_{+}$and the "tail" subtableau as $\tau_{-}$.

Now fix $i \geqslant 0$, and define a map $\phi: S_{i}(\lambda, \mu) \rightarrow S_{i}(\lambda, \bar{\mu})$ that is the identity on $\tau_{+}$ and which maps each $\tau_{-}$to $\bar{\tau}_{-}^{*}$. Notice that the "flipping" portion of $\left.\phi\right|_{\tau_{-}}$ensures that $\phi(\tau)$ has content $\bar{\mu}$. By Lemmas 8 and 9 we see that $\mathrm{n}_{\text {inv }}\left(\bar{\tau}^{*}\right)=j(N-j)-\mathrm{n}_{\text {inv }}(\bar{\tau})=$ $j(N-j)-j(N-j)+\mathrm{n}_{\text {inv }}(\tau)=\mathrm{n}_{\text {inv }}(\tau)$, ensuring that $\phi(\tau)$ is in fact an element of $S_{i}(\lambda, \bar{\mu})$. As $\phi$ is clearly reversible it represents a bijection.

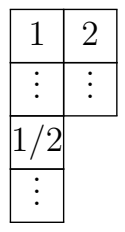

Figure 4: General form of a two-column row-standard tableau with content $\mu=1^{j} 2^{N-j}$

Theorem 11. Take any tableau shape $\lambda$ and any content $\mu=1^{\mu_{1}} 2^{\mu_{2}} \ldots M^{\mu_{M}}$ compatible with $\lambda$. For any permutation $\sigma \in S_{M}$, we have $\left|S_{i}(\lambda, \mu)\right|=\left|S_{i}(\lambda, \sigma(\mu))\right|$ for all $i \geqslant 0$.

Proof. We show $\left|S_{i}(\lambda, \mu)\right|=\left|S_{i}(\lambda, \sigma(\mu))\right|$ for a simple transposition $(a, a+1) \in S_{M}$ of consecutive elements $a, a+1 \in\{1,2, \ldots, M\}$. The general result then follows from repeated application of our procedure.

So take consecutive values $a, a+1 \in\{1,2, \ldots, M\}$. We begin by fixing semistandard $T \in S(\lambda, \mu)$ and identifying all instances of $a, a+1$ in $T$. The boxes with these two entries form a skew sub-tableau $\eta_{T}$ of $T$ with content $a^{\mu_{a}}(a+1)^{\mu_{a+1}}$. As $T$ is row-standard, no row in $\eta_{T}$ contains more than two entries. Subdivide $\eta_{T}$ into a set of "blocks" $\eta_{T}^{j}$, one for each upper-left corner entry $\alpha_{j}$ in $\eta_{T}$, by working leftward through the columns of $T$ and assigning to $\eta_{T}^{j}$ all entries that are below or to the right of $\alpha_{j}$ and which have not yet been assigned to any previous block. An example of this procedure is shown in Figure 1.

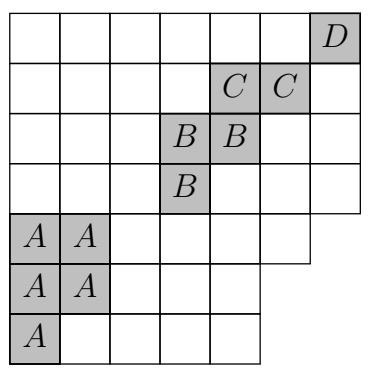

Figure 5: A standarized 7-row tableau with four "blocks" for the consecutive values $a, a+1$.

With the $\eta_{T}^{j}$ clearly defined, take any $\tau \in I(\lambda, \mu)$ such that $\operatorname{st}(\tau)=T$. Denote by $\eta_{\tau}^{j}$ (respectively $\eta_{\tau}$ ) the entries in $\tau$ that correspond to the entries of $\eta_{T}^{j}$ (respectively $\eta_{T}$ ) in 
$T$. Clearly, $\eta_{\tau}$ is merely the collection of all instances of $a$ and $a+1$ in $\tau$. If multiple instances of $a$ or $a+1$ appear in the same column of $\tau$, we ensure that each such instance is assigned a unique block $\eta_{\tau}^{j}$ by assuming that repeated instances of a fixed entry in the same column maintain the same relative height ordering as we pass between $\tau$ and $T$. We informally refer to each $\eta_{\tau}^{j}$ as an "inverted block".

Now consider the set of all semistandard tableaux $S(\lambda, \mu)$. Divide elements of $S(\lambda, \mu)$ into subsets depending upon the exact placement of their skew sub-tableau $\eta_{T}$, and for each distinct placement $\gamma$ define $S^{\gamma}(\lambda, \mu)=\left\{T \in S(\lambda, \mu) \mid \eta_{T}\right.$ has placement $\gamma$ in $\left.T\right\}$. For each $i \geqslant 1$, similarly divide all $i$-inverted tableaux $S_{i}(\lambda, \mu)$ into subsets based upon the placement of $\eta_{T}$ in their standardizations, so that $S_{i}^{\gamma}(\lambda, \mu)=\left\{\tau \in S_{i}(\lambda, \mu) \mid \operatorname{st}(\tau) \in\right.$ $\left.S^{\gamma}(\lambda, \mu)\right\}$. Pause to notice that, since $a$ and $a+1$ are consecutive numbers, the possible placements $\gamma$ are identical for $S(\lambda, \mu)$ and $S(\lambda, \sigma(\mu))$. To show $\left|S_{i}(\lambda, \mu)\right|=\left|S_{i}(\lambda, \sigma(\mu))\right|$, our strategy is to define a map $\phi: S_{i}(\lambda, \mu) \rightarrow S_{i}(\lambda, \sigma(\mu))$ that restricts to a bijection $\left.\phi\right|_{\gamma}: S_{i}^{\gamma}(\lambda, \mu) \rightarrow S_{i}^{\gamma}(\lambda, \sigma(\mu))$ for each possible placement $\gamma$.

So fix a placement $\gamma$ as well as an inversion number $i \geqslant 0$. To define our bijection, begin by recalling that an inverted tableau is uniquely identified by its standardization alongside a collection of column-specific inversion pairs (with multiplicities). For each $\tau \in S_{i}^{\gamma}(\lambda, \mu)$, we divide the $i$ inversion pairs of $\tau$ into subsets depending upon whether their participants both lie in a specific inverted block $\eta_{\tau}^{j}$. For each $j$, denote by $\zeta_{j}$ the set of all inversion pairs $\left(b_{1}, b_{2}\right)$ of $\tau$ such that $b_{1}, b_{2} \in \eta_{\tau}^{j}$. Then denote by $\widetilde{\zeta}$ the set of all inversion pairs of $\tau$ that aren't in $\zeta_{j}$ for any $j$.

Notice that each "block" $\eta_{T}^{j}$ of $T \in S^{\gamma}(\lambda, \mu)$ is a (non-skew) one- or two-column tableau with content of the form $a^{x}(a+1)^{y}$ for some $x, y \geqslant 0$. For any $\tau \in S_{i}^{\gamma}(\lambda, \mu)$ with $\operatorname{st}(\tau)=T$, specifying $\zeta_{j}$ allows us to associate each inverted block $\eta_{\tau}^{j}$ with a specific $\left|\zeta_{j}\right|$-inverted young tableau with content $a^{x}(a+1)^{y}$ whose standardization is $\eta_{T}^{j}$. Notice that these are precisely the sort of inverted tableau addressed in Lemma 10.

We are now ready to define our map $\left.\phi\right|_{\gamma}: S_{i}^{\gamma}(\lambda, \mu) \rightarrow S_{i}^{\gamma}(\lambda, \sigma(\mu))$. For an arbitrary $\tau \in S_{i}^{\gamma}(\lambda, \mu)$, we define $\left.\phi\right|_{\gamma}(\tau)$ as follows:

1. For each inverted block $\eta_{\tau}^{j}$ of $\tau$, we interpret $\eta_{\tau}^{j}$ as an $\left|\zeta_{j}\right|$-inverted tableau with content $a^{x}(a+1)^{y} .\left.\quad \phi\right|_{\gamma}$ is then defined on $\eta_{\tau}^{j}$ using the bijection of Lemma 10, replacing $\eta_{\tau}^{j}$ with a new inverted block $\eta_{\phi(\tau)}^{j}$ that we interpret as a $\left|\zeta_{j}\right|$-inverted tableau of the same shape but with content $(a+1)^{x} a^{y}$. On the level of inversion pairs, this simply swaps $\zeta_{j}$ for $\left|\zeta_{j}\right|$ inversion pairs whose members lie within $\eta_{\phi(\tau)}^{j}$.

2. Outside of $\eta_{\tau},\left.\phi\right|_{\gamma}$ is the identity apart from entries that lie to the left of a block $\eta_{\tau}^{j}$. If the bijection of Lemma 10 changes the height order of entries directly to the left of $\eta_{\tau}^{j}$, permute the "partial rows" to the left of $\eta_{\tau}^{j}$ in the unique way that preserves the height order of those elements. On the level of inversion pairs, this is the unique rearrangement of entries outside of $\eta_{\tau}$ so that the $|\widetilde{\zeta}|$ is unchanged as we pass from $\tau$ to $\left.\phi\right|_{\gamma}(\tau)$ (specific inversion pairs in $\widetilde{\zeta}$ may only change in that they swap instances of $a$ and $a+1$, as determined by the bijection of Lemma 10 . 
For an example of the procedure above, see Figure 6. Our map $\left.\phi\right|_{\gamma}$ is clearly welldefined. When considered on the level of inversion pairs, the map also fixes the total number of inversion pairs. As Step \#2 fixes content and Step \#1 flips the content of $a$ and $a+1$, as per Lemma 10, the output always has content $\sigma(\mu)$. To conclude that $\left.\phi\right|_{\gamma}$ is our desired map it remains to be shown that $\left.\phi\right|_{\gamma}(\tau)$ is always row-standard and hence in $S_{i}^{\gamma}(\lambda, \mu)$, as well as that $\left.\phi\right|_{\gamma}$ is bijective.

The fact that $\left.\phi\right|_{\gamma}(\tau)$ is always row-standard follows from our definition of the $\eta_{T}^{j}$ and the $\eta_{\tau}^{j}$. As $a$ and $a+1$ are consecutive numbers, reassigning entries within $\eta_{\tau}$ never results in an instance of $a$ or $a+1$ appearing leftward of an entry less than $a$, and similarly never results in an instance of of $a$ or $a+1$ appearing rightward of an entry greater than $a+1$. Seeing as the bijection of Lemma 10 preserves the fact that the rightward column of each inverted block consists solely of $a+1$ entries, it is impossible for an instance of $a+1$ from a fixed inverted block $\eta_{\tau}^{j}$ to end up left of an instance of $a$ from that same inverted block. Lastly, due to the way that all "boundary entries" in $\eta_{T}$ are assigned to the leftmost of the two adjacent blocks (such as the lower-right corner in Figure 5 that is assigned a $B$ instead of a $C$ ), a valid rearrangement of entries within $\eta_{\tau}$ never results in an instance of $a+1$ from one inverted block lying to the left of an instance of $a$ from another inverted block. This is because those boundary entries are always necessarily an instance of $a+1$. These observations combine to let us conclude that $\left.\phi\right|_{\gamma}(\tau)$ is row-standard for any $\tau \in S_{i}^{\gamma}(\lambda, \mu)$, and hence that $\left.\phi\right|_{\gamma}(\tau) \in S_{i}^{\gamma}(\lambda, \sigma(\mu))$.

To conclude that $\left.\phi\right|_{\gamma}$ is a bijection, we again cite the fact that an inverted tableaux is uniquely determined by its standardization along with a collection of column-specific inversion pairs. If $\tau_{1}, \tau_{2} \in S_{i}^{\gamma}(\lambda, \mu)$ differ in that their inversion pair subsets $\zeta_{j}$ are distinct for some $j$, the bijection of Lemma 10 ensures that $\left.\phi\right|_{\gamma}\left(\tau_{1}\right),\left.\phi\right|_{\gamma}\left(\tau_{2}\right)$ have distinct sets of inversion pairs in their $j^{t h}$ block. If $\tau_{1}, \tau_{2} \in S_{i}^{\gamma}(\lambda, \mu)$ differ in that their inversion pair subsets $\widetilde{\zeta}$ are distinct, the fact that Step \#2 of our bijection fixes $\widetilde{\zeta}$ ensures that $\left.\phi\right|_{\gamma}\left(\tau_{1}\right),\left.\phi\right|_{\gamma}\left(\tau_{2}\right)$ have distinct subsets $\widetilde{\zeta}$. It follows that $\left.\phi\right|_{\gamma}$ is injective. The fact that $\left.\phi\right|_{\gamma}$ is surjective quickly follows from the fact that the bijection of Lemma 10 is surjective and, since $a$ and $a+1$ are consecutive values, there is a bijection between acceptable subsets of inversion pairs $\widetilde{\zeta}$ for elements of $S_{i}^{\gamma}(\lambda, \mu)$ and acceptable subsets $\widetilde{\zeta}$ for elements of $S_{i}^{\gamma}(\lambda, \sigma(\mu))$. Thus we conclude that $\left.\phi\right|_{\gamma}: S_{i}^{\gamma}(\lambda, \mu) \rightarrow S_{i}^{\gamma}(\lambda, \sigma(\mu))$ is our desired map.

As one quick corollary of Theorem 11, notice that the total number of inverted semistandard tableaux of shape $\lambda$, when ranging over all possible numbers of inversions, is also invariant under permutation of content:

Corollary 12. Take any tableau of shape $\lambda$ and any content $\mu=1^{\mu_{1}} 2^{\mu_{2}} \ldots K^{\mu_{K}}$ compatible with $\lambda$. For any permutation $\sigma \in S_{M},|I(\lambda, \mu)|=|I(\lambda, \sigma(\mu))|$.

\section{Enumeration of Inverted Semistandard Young Tableaux}

With the tools of Section 2 in place, we are ready to present enumerative results about inverted semistandard Young tableaux. As with [1], closed formulas for $\left|S_{i}(\lambda, \mu)\right|$ when $i$ is arbitrary are only tractable for certain "easy" choices of $\lambda$, namely one-column and 


\begin{tabular}{|c|c|c|c|c|c|c|c|c|c|c|c|c|c|c|c|}
\hline 1 & 4 & 6 & 8 & 1 & 4 & 7 & 8 & 1 & 4 & 6 & 7 & 1 & 4 & 6 & 7 \\
\hline 2 & 5 & 6 & 7 & 2 & 5 & 6 & 7 & 2 & 5 & 6 & 8 & 2 & 5 & 7 & 8 \\
\hline 3 & 7 & 8 & 9 & 4 & 7 & 8 & 9 & 2 & 6 & 8 & 9 & 2 & 6 & 8 & 9 \\
\hline 2 & 6 & 10 & 11 & 3 & 7 & 10 & 11 & 3 & 6 & 10 & 11 & 3 & 7 & 10 & 11 \\
\hline 4 & 6 & 11 & 12 & 2 & 6 & 11 & 12 & 4 & 7 & 11 & 12 & 4 & 7 & 11 & 12 \\
\hline
\end{tabular}

Figure 6: Part of the bijection $\phi: S_{5}(\lambda, \mu) \rightarrow S_{5}(\lambda, \sigma(\mu))$ from the proof of Theorem 11 , for $\lambda=(4,4,4,4,4), \mu=1^{1} 2^{2} 3^{1} 4^{2} 5^{1} 6^{4} 7^{2} 8^{2} 9^{1} 10^{1} 11^{2} 12^{1}$ and $\sigma=(67)$. The left side shows $\tau$ and $\sigma(\tau)$, both of which have inversion pairs $(3,4)^{1},(4,5)^{2},(6,7)^{2},(6,7)^{2},(7,8)^{4}$. The right side shows $\operatorname{st}(\tau)$ and $\operatorname{st}(\sigma(\tau))$. Notice how the first column of $\sigma(\tau)$ has been reordered to the left of the bottom "block" in order to maintain height order.

one- or two-row shapes. After fully addressing those "easy" cases, we directly enumerate $S_{1}(\lambda, \mu)$ for arbitrary $\lambda$ and $\mu$ by placing that set in bijection with a collection of sets of (non-inverted) semistandard Young tableaux $\bigcup_{k} S_{0}\left(\widetilde{\lambda}_{k}, \mu\right)$, thus generalizing Theorems 3.1 and 3.2 of [1].

\subsection{Enumerating $i$-Inverted Semistandard Young Tableaux, $\lambda=(1,1, \ldots, 1)$}

In the standard tableaux setting of [1], one of the few choices of $\lambda$ for which specific $\left|S_{i}(\lambda)\right|$ could be directly computed were the single-column shapes $\lambda=1^{m}(m \geqslant 1)$. As inverted standard Young tableaux with one-column are equivalent to permutations, that paper cited the standard result [8] to give $\left|S_{i}(\lambda)\right|=M(m-1, i)$, where $M(m-1, i)$ is the Mahonian number. If we let $[p]_{q}=1+q+\ldots+q^{p-1}$ be the q-number and let $[p]_{q} !=[1]_{q}[2]_{q} \ldots[p]_{q}$ be the q-factorial, this gave the generating function:

$$
\sum_{i=0}^{\infty}\left|S_{i}(\lambda)\right| q^{i}=(1+q)\left(1+q+q^{2}\right) \ldots\left(1+q+\ldots+q^{m-1}\right)=[2]_{q}[3]_{q} \ldots[m]_{q}=[m]_{q} \text { ! }
$$

The single-column case is also relatively tractable when we allow for repeated entries, yielding a direct generalization of the generating function from Equation 3. In what follows, we use the standard notation $\left(\begin{array}{l}a \\ b\end{array}\right)_{q}=\frac{[a]_{q} !}{[b]_{q} ![a-b]_{q} !}=\frac{\left(1-q^{a}\right)\left(1-q^{a-1}\right) \ldots\left(1-q^{a-b+1}\right)}{(1-q)\left(1-q^{2}\right) \ldots(1-q)^{b}}$ for the q-binomial coefficients.

Theorem 13. Let $\lambda=1^{M}$ be the one-column tableau shape with $M$ total entries, and let $\mu=1^{\mu_{1}} 2^{\mu_{2}} \ldots M^{\mu_{m}}$ be some content such that $\sum_{k} \mu_{k}=M$. Then we have generating function:

$$
\sum_{i=0}^{\infty}\left|S_{i}(\lambda, \mu)\right| q^{i}=\left(\begin{array}{c}
\mu_{1} \\
\mu_{1}
\end{array}\right)_{q}\left(\begin{array}{c}
\mu_{1}+\mu_{2} \\
\mu_{2}
\end{array}\right)_{q} \ldots\left(\begin{array}{c}
M \\
\mu_{m}
\end{array}\right)_{q}=\frac{[M]_{q} !}{\left[\mu_{1}\right]_{q} !\left[\mu_{2}\right]_{q} ! \ldots\left[\mu_{m}\right]_{q} !}
$$

Proof. It is possible to "build up" any inverted tableau $\tau \in I(\lambda, \mu)$ by recursively inserting $\mu_{n}$ copies of $n$ into a one-column tableau $\tau_{n-1}$ with content $1^{\mu_{1}} 2^{\mu_{2}} \ldots(n-1)^{\mu_{n-1}}$, producing a sequence $\left\{\tau_{1}, \tau_{2}, \ldots, \tau_{m}\right\}$ of one-column tableaux such that $\tau_{m}=\tau$. Notice that distinct 
placements at any step in this process always results in distinct $\tau$, so this procedure describes a way to uniquely determine every element of $I(\lambda, \mu)$. Furthermore, the number of inversion pairs in the resulting $\tau$ whose larger element is $n$ is determined entirely by the insertion of the $\mu_{n}$ copies of $n$ into $\tau_{n-1}$ : this "level $n$ step" is the only point at which such inversion pairs may appear, and the number of such inversion pairs is not dependent upon the prior arrangement of the $1^{\mu_{1}} 2^{\mu_{2}} \ldots(n-1)^{\mu_{n-1}}$ or the later placement of larger entries.

So fix a one-column tableau $\tau_{n-1}$ with content $1^{\mu_{1}} 2^{\mu_{2}} \ldots(n-1)^{\mu_{n-1}}$. A copy of $n$ placed above $j$ entries in $\tau_{n-1}$ results in $j$ inversion pairs whose larger entry is that instance of $n$. In particular, each instance of $n$ may be involved in up to $\mu_{1}+\mu_{2}+\ldots+\mu_{n-1}$ inversion pairs where it is the larger entry, and the number of such inversion pairs involving a particular instance of $n$ is independent of the placement of other instances of $n$. Now consider the number of tableaux obtained from $\tau_{n-1}$ with precisely $i$ inversion pairs whose larger entry is $n$. By our preceding comments, these tableaux are in bijection with partitions of $i$ into at most $\mu_{n}$ parts (one part corresponding to each instance of $n$ ) where each part is less than or equal to $\mu_{1}+\ldots+\mu_{n-1}$.

It is well known that the coefficient of $q^{i}$ in $\left(\begin{array}{c}a+b \\ a\end{array}\right)_{q}$ equals the number of partitions of $i$ into at most $a$ parts, with each part less than or equal to $b$. If $\left|\tau_{n-1}^{i}\right|$ denotes the number tableaux $\tau_{n}$ obtained from $\tau_{n-1}$ with precisely $i$ inversion pairs whose larger entry is $n$, we then have generating function

$$
\sum_{i=0}^{\infty}\left|\tau_{n-1}^{i}\right| q^{i}=\left(\begin{array}{c}
\mu_{1}+\mu_{2}+\ldots+\mu_{n} \\
\mu_{n}
\end{array}\right)_{q}
$$

As every inversion pair in $\tau \in I(\lambda, \mu)$ appears at a unique step in the sequence $\left\{\tau_{1}, \tau_{2}, \ldots, \tau_{m}\right\}$, multiplying the generating functions of Equation 4 for $1 \leqslant i \leqslant m$ gives the result.

Notice that if $\mu_{k}=1$ for all $k$, Theorem 13 recovers the standard tableaux result of Equation 3. Specialization of Theorem 13 at $q=1$ shows that the total number of inverted semistandard Young tableaux of shape $\lambda$ is $|I(\lambda, \mu)|=\frac{M !}{\mu_{1} ! \mu_{2} ! \ldots \mu_{m} !}$, independently verifying Proposition 6.

\subsection{Enumerating $i$-Inverted Semistandard Young Tableaux, $\lambda=(n, n)$}

The other basic shapes $\lambda$ that admitted a direct enumeration of i-inverted standard Young tableaux in [1] were one-row and two-row tableau shapes. The row-standard condition made one-row shapes predictably trivial: if $\lambda=(n)$ for any $n \geqslant 1$, then $\left|S_{0}(\lambda)\right|=1$ and $\left|S_{i}(\lambda)\right|=0$ for all $i \geqslant 1$. The two-row case involved a recognition of the fact that any tworow inverted tableau necessarily "split" after a column in which it possessed an inversion pair. As shown in [1], if $\lambda=(n, n)$ the formula for $\left|S_{i}(\lambda)\right|$ depends upon summations of products of Catalan numbers $C_{k}$ where the subscripts in each term partition $n$ : 


$$
\left|S_{i}(\lambda)\right|=\left(\sum_{k_{1}+\ldots+k_{i}=n} C_{k_{1}} C_{k_{2}} \ldots C_{k_{i}}\right)+\left(\sum_{l_{1}+\ldots+l_{i+1}=n} C_{l_{1}} C_{l_{2}} \ldots C_{l_{i+1}}\right)
$$

Equation 5 admits a very direct generalization to the semistandard case in the form of Theorem 14. This theorem also marks our first usage of Theorem 11 as a powerful simplifying tool:

Theorem 14. Let $\lambda=(n, n)$, any $n \geqslant 1$, and let $\mu=1^{\mu_{1}} 2^{\mu_{2}} \ldots$ be some content such that $\sum_{k} \mu_{k}=2 n$.

1. If $\mu_{k}>2$ for any $k$, then $\left|S_{i}(\lambda, \mu)\right|=0$ for all $i \geqslant 0$.

2. If $\mu_{k}=2$ for precisely $m$ choices of $k$ and $\mu_{k}=1$ for the remaining $2 n-2 m$ choices of $k$, then:

$$
\left|S_{i}(\lambda, \mu)\right|=\left(\sum_{j_{1}+\ldots+j_{i}=n-m} C_{j_{1}} C_{j_{2}} \ldots C_{j_{i}}\right)+\left(\sum_{l_{1}+\ldots+l_{i+1}=n-m} C_{l_{1}} C_{l_{2}} \ldots C_{l_{i+1}}\right)
$$

Where $C_{j}$ is the $j^{\text {th }}$ Catalan number, and the summations run over all ordered partitions of length $i$ and $i+1$, respectively.

Proof. Case \#1 is immediate because no such tableau can be row-standard. For Case \# 2 , by Theorem 11 we may assume that $\mu_{k}=2$ for $1 \leqslant k \leqslant m$ and $\mu_{k}=1$ for $k>m$. This means that the first $m$ columns of any $\tau \in I(\lambda, \mu)$ each consist of two instances of the same entry, and hence cannot partake in an inversion pair. Thus the only place where $\tau$ isn't predetermined, as well as the only place where $\tau$ may possess inversion pairs, is over it's final $n-m$ columns. Notice that, when restricted to these final $n-m$ columns, any $\tau \in I(\lambda, \mu)$ becomes a inverted standard Young tableau with $2(n-m)$ distinct entries $m+1, m+2, \ldots, 2 n-m$. If we define $\widetilde{\lambda}=(n-m, n-m)$, a truncation of $\tau \in I(\lambda, \mu)$ to its final $n-m$ columns and then a reindexing of its entries yields a bijection between $S_{i}(\lambda, \mu)$ and $S_{i}(\widetilde{\lambda})$ for all $i \geqslant 0$. The result then follows from Theorem 2.3 of [1].

If $\mu_{k}=1$ for all $k$, the formula of Theorem 14 very obviously simplifies to the standard tableaux formula of Equation 5. Less obvious from Theorem 14 is an enumeration of the total number of inverted tableaux $|I(\lambda, \mu)|$, but a similar bijection with shorter two-row standard tableaux yields the following:

Proposition 15. Let $\lambda=(n, n)$ for any $n \geqslant 1$, and let $\mu=1^{\mu_{1}} 2^{\mu_{2}} \ldots$ be some content with $\sum_{k} \mu_{k}=2 n$. If $\mu_{k}=2$ for precisely $m$ choices of $k(0 \leqslant m \leqslant n)$ and $\mu_{k}=1$ otherwise, then $|I(\lambda, \mu)|=\left(\begin{array}{c}2(n-m) \\ n-m\end{array}\right)$. 
Proof. By Theorem 12, we may assume that $\mu_{k}=2$ for $1 \leqslant k \leqslant m$ and $\mu_{k}=1$ for $k>m$. As in the proof of Theorem 14, this means that the first $m$ columns of any $\tau \in I(\lambda, \mu)$ each consist of two instances of the same entry, and that elements of $I(\lambda, \mu)$ are in bijection with inverted standard Young tableaux of shape $\tilde{\lambda}=(n-m, n-m)$. The result then follows from Proposition 2.1 of [1], as we merely need to specify which of the $2(n-m)$ non-repeated entries appear in the first row.

\subsection{Enumerating 1-Inverted Semistandard Young Tableaux}

Enumeration of $S_{i}(\lambda, \mu)$ for general $\lambda$ and any $i \geqslant 0$ is a daunting (and potentially intractable) task that wasn't even accomplished in the non-repeated entry case of [1]. If one wishes to address arbitrary $\lambda$, one enumeration $\left|S_{i}(\lambda, \mu)\right|$ that remains approachable is the single inversion pair case of $i=1$. In this subsection we exhibit a bijection between 1-inverted semistandard Young tableaux and (0-inverted) semistandard Young tableaux of a collection of related shapes. Unlike in [1], this doesn't allow for an immediate determination of $\left|S_{1}(\lambda, \mu)\right|$ via the hook-length formula, seeing as the hook-content formula for semistandard Young tableaux is ill-suited to enumeration of tableaux with a specific content. Nonetheless, it does replace the set $S_{1}(\lambda, \mu)$ with a far better understood collection of sets $S_{0}(\widetilde{\lambda}, \mu)$. The author also conjectures that the approach of Theorems 16 and 17 may be modified for the calculation of $\left|S_{i}(\lambda, \mu)\right|$ for some $i>1$, akin to Conjecture 4.4 in $[1]$.

Theorem 16. Let $m, n \geqslant 1$, and consider the $m$-row shapes $\lambda=(n, \ldots, n), \widetilde{\lambda}=(n+$ $1, n, \ldots, n, n-1)$. For any content $\mu$ compatible with $\lambda,\left|S_{1}(\lambda, \mu)\right|=\left|S_{0}(\tilde{\lambda}, \mu)\right|$.

If $\lambda$ is a rectangular shape of size $m \times n$, then $\widetilde{\lambda}$ is the "stair-step" shape formed by moving the the lower-right corner in the Young diagram of shape $\lambda$ to a new, $(n+1)^{s t}$ column. See Figure 7 for an example of this shape change. Also pause to notice that this is the only way to rearrange the boxes in a Young diagram of shape $\lambda$ to produce another valid Young diagram whereby the old lower-right corner in $\lambda$ has been moved to a new lower-right corner in a higher row.

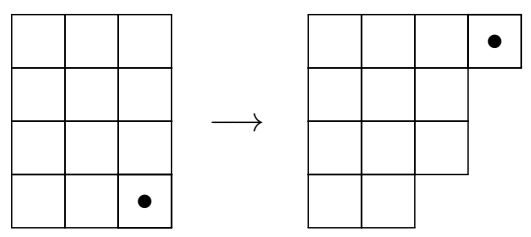

Figure 7: Shape change in the bijection of Theorem 16 for $\lambda=(3,3,3,3)$

Proof of Theorem 16. As in Theorem 3.1 of [1], we define two functions $\phi_{1}: S_{1}(\lambda, \mu) \rightarrow$ $S_{0}(\widetilde{\lambda}, \mu), \phi_{2}: S_{0}(\widetilde{\lambda}, \mu) \rightarrow S_{1}(\lambda, \mu)$ such that $\phi_{1}$ and $\phi_{2}$ are inverses of one another. The general outline of both procedures is in line with the "repeated bumping" maps defined in [1], apart from the addition of conditions that address how bumping behaves in the vicinity of repeated entries. 
For the map $\phi_{1}: S_{1}(\lambda, \mu) \rightarrow S_{0}(\widetilde{\lambda}, \mu)$, take $\tau \in S_{1}(\lambda, \mu)$ and identify the sole inversion pair $(a, b)$ of $\tau$. Assume that $(a, b)$ appears in the $k^{t h}$ column of $\tau$; as $(a, b)$ is the tableau's only inversion pair it must be the case that $b$ appears immediately above $a$ in the $k^{\text {th }}$ column. Let $b=c_{k}$. Our strategy is to recursively "bump" a sequence of elements $\left\{c_{k}, c_{k+1}, \ldots, c_{n}\right\}$, one from each column of $\tau$ beginning with the $k^{t h}$ column, rightward by one column each. Our procedure is defined as follows:

1. Beginning at the site of the inversion pair $(a, b)$ in the $k^{\text {th }}$ column, let $c_{k}=b$. If there are any columns in $\tau$ to the left of the $k^{\text {th }}$, reorder those columns so that they are each non-increasing from top-to-bottom. This reordering guarantees that no new inversion pairs will be added in leftward columns due to the elimination of the $(a, b)$ inversion pair.

2. At the $j^{\text {th }}$ column in the procedure, if $j<n$ let $c_{j+1}$ be the smallest entry in the $(j+1)^{\text {st }}$ column such that $c_{j+1}>c_{j}$. Then move $c_{j}$ to the spot occupied by $c_{j+1}$, temporarily allowing two entries in that spot. This leaves an empty box in the $j^{\text {th }}$ column where $c_{j}$ was formerly located. Recursively fill any open spots in the $j^{\text {th }}$ column by moving the smaller of the two entries directly below or directly to the right of the empty box into that box. If both entries are equal at any step in this process, move the entry directly to the right of the empty box into that box. Repeat this procedure until the empty box has been moved into the $(j+1)^{s t}$ column.

3. At the $j^{\text {th }}$ column in the procedure, if $j=n$ move $c_{n}$ to the top spot of a new $(n+1)^{s t}$ column. Then slide all entries that were below $c_{n}$ in the $n^{\text {th }}$ column up by one spot.

An example of the full procedure is shown in Figure 8. Notice that this procedure always results in a tableau of the correct shape $\widetilde{\lambda}$, and that the resulting tableau $\widetilde{\lambda}$ lacks inversion pairs because the procedure is defined to ensure that every column is nonincreasing from top-to-bottom. The "forward bumping" and "back sliding" procedures are also defined to ensure that the resulting tableau is row-standard. In particular, observe that the condition in \#2 whereby the rightward of two identical entries is slid left prevents two identical entries from appearing in the same row. As every step in the procedure is uniquely determined, we may conclude that the procedure defines a well-defined function $\phi_{1}: S_{1}(\lambda, \mu) \rightarrow S_{0}(\widetilde{\lambda}, \mu)$.

For the map $\phi_{2}: S_{0}(\tilde{\lambda}, \mu) \rightarrow S_{1}(\lambda, \mu)$, take $T \in S_{0}(\tilde{\lambda}, \mu)$ and define $c_{n}$ to be the sole entry in the $(n+1)^{s t}$ column of $T$. Our strategy is to define a sequence of entries $\left\{c_{n}, c_{n-1}, \ldots\right\}$, where $c_{j}$ begins in the $(j+1)^{s t}$ column of $T$, and then recursively "reverse bump" $c_{j}$ into the $j^{\text {th }}$ column in a manner that reverses the $\phi_{1}$ procedure between any two columns. Our new procedure at the $j^{\text {th }}$ column is as follows:

1. Consider $c_{j}$, which begins in the $(j+1)^{s t}$ column. There is guaranteed to be an empty box in the $j^{\text {th }}$ column. Recursively fill that empty box with the largest entry from among $c_{j}$, the entry directly above the empty box, and the entry directly to the 


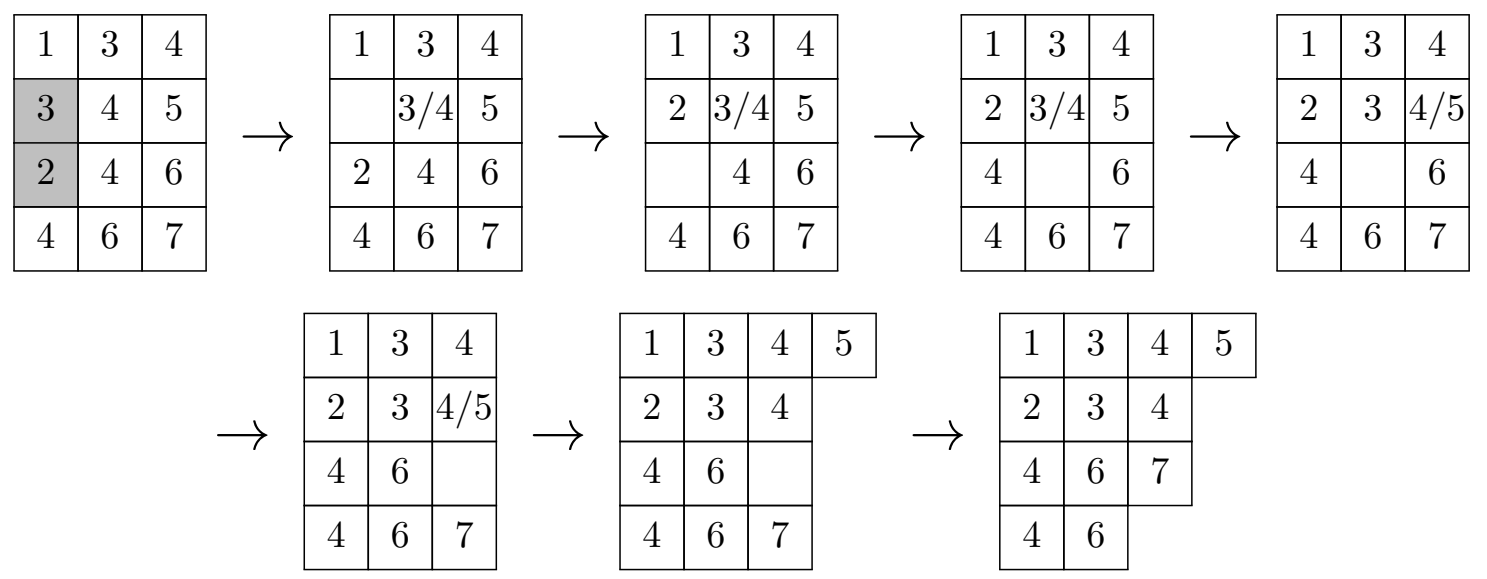

Figure 8: Part of the $S_{1}(\lambda, \mu) \hookrightarrow S_{0}(\widetilde{\lambda}, \mu)$ bijection for $\lambda=(3,3,3,3)$ and $\mu=$ $1^{1} 2^{1} 3^{2} 4^{4} 5^{1} 6^{2} 7^{1}$

left of the empty box. If the largest value at any point in this process is shared by two or more of those three entries, preference is given to entries that begin in a more leftward position. Repeat this procedure until the empty box is moved leftward to the $(j-1)^{s t}$ column or $c_{j}$ directly fills the empty box.

2. If the empty box in the $j^{\text {th }}$ column is moved leftward to the $(j-1)^{s t}$ column, define $c_{j-1}$ to be the largest entry in the $j^{t h}$ column that is strictly less than $c_{j}$. Move $c_{j}$ in the box occupied by $c_{j-1}$, temporarily producing a box with two entries, and then repeat step \#1 with $c_{j-1}$ and the $(j-1)^{s t}$ column.

3. If the empty box in the $j^{\text {th }}$ column is directly filled by $c_{j}$, we introduce a single new inversion pair $\left(a, c_{j}\right)$ in the $j^{t h}$ column with $c_{j}$ and the entry lying directly above the box into which $c_{j}$ was inserted. Do this by flipping the rows containing $c_{j}$ and $a$ from the $j^{\text {th }}$ column leftward. Flipping leftward entries along with $c_{j}$ and $a$ ensures that no additional inversion pairs are added in leftward columns.

For an example of this second procedure, see Figure 9. Notice that the "sliding" and "reverse bumping" rules of steps \#1 and \#2, along with the addendum addressing when identical entries are involved, ensure that the tableau is row-standard and columnsemistandard. This means that the only inversion in the resulting tableau is the one introduced in step \#3. Also notice that the resulting tableaux always admits an inversion at this spot in the $j^{\text {th }}$ column since $c_{j}<c_{j+1}$ and our procedure ensures that the entry $c_{j}$ always drops down at least one row at this final step (see the proof of Theorem 3.1 in [1] for additional discussion of this final fact). As every step in the procedure is uniquely determined, we conclude that the procedure induces a well-defined function $\phi_{2}: S_{0}(\widetilde{\lambda}, \mu) \rightarrow S_{1}(\lambda, \mu)$.

Our maps $\phi_{1}$ and $\phi_{2}$ have been constructed so that they are inverses of one another. In particular, the intermediate steps of the two procedures coincide after each column. This holds true even if specific "bumps" / "reverse bumps" aren't direct reversals of 


\begin{tabular}{|c|c|c|c|c|c|c|c|c|c|c|c|c|c|}
\hline 1 & 2 & 4 & 5 & \multirow{3}{*}{$\rightarrow$} & 1 & 2 & \multirow{2}{*}{\multicolumn{2}{|c|}{$\frac{4}{5}$}} & \multirow{3}{*}{$\rightarrow$} & \multicolumn{2}{|c|}{1} & \multicolumn{2}{|c|}{\begin{tabular}{l|l}
2 & $4 / 5$
\end{tabular}} \\
\hline 2 & 4 & \multirow[t]{2}{*}{5} & & & 2 & 4 & & & & & 4 & & 5 \\
\hline 3 & 6 & & & & 3 & & 6 & & & & & & 6 \\
\hline & 1 & 2 & $4 / 5$ & & 1 & & & 5 & & 2 & 4 & 5 & \\
\hline$\rightarrow$ & 2 & & 5 & $\rightarrow$ & 2 & & & 5 & $\rightarrow$ & 1 & 2 & 5 & \\
\hline & 3 & 4 & 6 & & 3 & & & 6 & & 3 & 4 & 6 & \\
\hline
\end{tabular}

Figure 9: Part of the $S_{0}(\widetilde{\lambda}, \mu) \hookrightarrow S_{1}(\lambda, \mu)$ bijection for $\lambda=(3,3,3), \mu=1^{1} 2^{2} 3^{1} 4^{2} 5^{2} 6^{1}$. Notice how the first column has been reordered at the final step to preserve its height ordering.

one another when working within a specific column, as demonstrated by an inability to directly reverse specific steps in the examples of Figure 8 or Figure 9 . Since $\phi_{2} \circ \phi_{1}(\tau)=\tau$ for all $\tau \in S_{1}(\lambda, \mu)$ and $\phi_{1} \circ \phi_{2}(T)=T$ for all $T \in S_{0}(\widetilde{\lambda}, \mu)$, we may deduce that both maps are bijections and that $\left|S_{1}(\lambda, \mu)\right|=\left|S_{0}(\tilde{\lambda}, \mu)\right|$.

Theorem 16 admits a generalization to non-rectangular tableaux that utilizes slight modifications of the procedures for $\phi_{1}$ and $\phi_{2}$. As with Theorem 3.2 of [1], this requires the introduction of additional terminology that describes the resulting shape change in the tableaux.

So consider the tableau shape $\lambda=\left(\lambda_{1}, \ldots, \lambda_{m}\right)$. Define $d_{i}=\lambda_{i}-\lambda_{i+1}$ for $1 \leqslant i<m$ and $d_{m}=\lambda_{m}$, meaning that $d_{i}>0$ if the $i^{\text {th }}$ row contains a "lower-right corner" and $d_{i}=0$ otherwise. Then define $\widetilde{d}_{i}=\lambda_{i-1}-\lambda_{i}$ for $1<i \leqslant m$ and $\widetilde{d}_{1}=\infty$, so that $\widetilde{d}_{i}>0$ if and only if an additional box may be added to the $i^{\text {th }}$ row without yielding an invalid tableau shape. With $d_{i}$ and $\widetilde{d}_{i}$ defined for each row of the tableau shape $\lambda$, we have the following:

Theorem 17. Consider the tableau shape $\lambda=\left(\lambda_{1}, \ldots, \lambda_{m}\right)$ with $m>1$, and let $\mu$ be any content compatible with $\lambda$. Then:

$$
\left|S_{1}\left(\left(\lambda_{1}, \ldots, \lambda_{m}\right), \mu\right)\right|=\sum_{E}\left|S_{0}\left(\left(\lambda_{1}+\epsilon_{1}, \ldots, \lambda_{m}+\epsilon_{m}\right), \mu\right)\right|
$$

Where the summation is over all tuples $E=\left(\epsilon_{1}, \ldots, \epsilon_{m}\right)$ such that $\epsilon_{i}=0, \pm 1$ for all $i$, $\epsilon_{i}=-1$ for precisely one $i=i_{1}$ with $i_{1}>1$ and $d_{i_{1}}>0$, and $\epsilon_{i}=1$ for precisely one $i=i_{2}$ with $i_{2}<i_{1}$ and $\widetilde{d}_{i_{2}}>0$.

Proof. Follows directly from the procedures of Theorem 16, as with the proof of Theorem 3.2 of [1]. For an example of the shape change in this general bijection, which involves identifying all possible ending points of the $\phi_{1}$ procedure from the proof of Theorem 16, see Figure 10. 


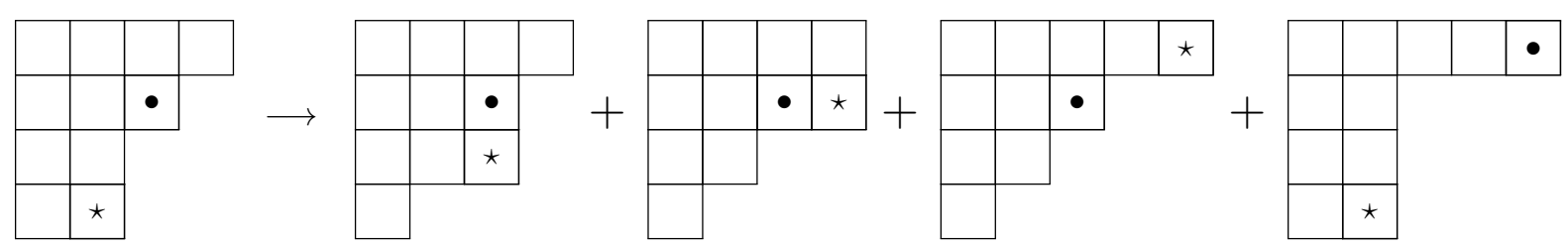

Figure 10: Shape change in the bijection of Theorem 17 when $\lambda=(4,3,2,2)$

\section{References}

[1] Jonathan E. Beagley and Paul Drube, Combinatorics of tableau inversions, Elec. J. of Combin. 22 (2) (2015).

[2] Paul Drube, $\mathfrak{s l}_{3}$ skein modules of surfaces, in preparation.

[3] Lucas Fresse, Betti numbers of Springer fibers in type A, J. Algebra 322 (2009), 2566-2579.

[4] Lucas Fresse, Ronit Mansour and Anna Melnikov, Unimodality of the distribution of Betti numbers for some Springer fibers, J. Algebra 391 (2013), 284-304.

[5] William Fulton, Young tableaux, with applications to representations theory and geometry, Cambridge University Press, 1996.

[6] Heather M. Russell, The Bar-Natan skein module of the solid torus and the homology of $(n, n)$ Springer varieties, Geometriae Dedicata 142 (2009), 71-89.

[7] Michael Shynar, On inversions in standard Young tableaux, Formal Power Series and Algebraic Combinatorics (2004), 241-248.

[8] Richard Stanley, Enumerative combinatorics vol. 2, Cambridge University Press, 1999.

[9] Juliana Tymoczko, A simple bijection between standard $3 \times n$ tableaux and irreducible webs for $s_{3}$, J. Algebraic Combin. 35 (2012), 611-632. 